\title{
Anesthesia for an emergency gastrorrhagia operation in a patient suspected with COVID-19: a case report
}

\section{Yu Chen}

Chinese PLA general Hospital https://orcid.org/0000-0003-2545-794X

Hao Lv (D mzk6plagh@163.com )

Chinese PLA general Hospital https://orcid.org/0000-0002-0175-6120

\section{Case Report}

Keywords: suspected case, COVID-19, preventive measures, anesthesia

Posted Date: July 21st, 2020

DOI: https://doi.org/10.21203/rs.3.rs-44673/v1

License: (c) (1) This work is licensed under a Creative Commons Attribution 4.0 International License. Read Full License 


\section{Abstract}

Background

The spread of the Coronavirus Disease 2019 (COVID-19) in China since December 2019 was very grave. According to Chinese govenment's guidelines, patients can be divided into confirmed cases and suspected cases. The protective measures for both are the same.

Case summary

We report a case of an emergency gastrorrhagia operation for a patient suspected to have contracted COVID-19. A 54-year-old man with massive gastric hemorrhage was scheduled for an emergency operation for exploratory hemostasis. COVID-19 infection, however, could not be ruled out, and the patient was treated as a suspected case. All the protective measures were based on patients infected or suspected to be infected with COVID-19. Before the operation, we carried out a series of protective measures, such as preparing the operating room and wearing personal protective gear. We increased the dose of neuromuscular blocking agents during the induction of anesthesia to prevent the splashing of secretions by coughing. The operation went smoothly, and the patient was transferred to an intensive care unit (ICU) in the isolation ward for extubation. Seven days later, the patient was ruled out for COVID-19 infection.

Conclusion

The patient recovered successfully from the massive gastric hemorrhage, and the medical staff were not infected.

\section{Introduction}

COVID-19 broke out in China in December 2019 and then spread rapidly through the rest of the world. The first laboratory-confirmed case of COVID-19 was reported in Wuhan on December 8, 2019. Until March 28, 2020, 82,278 people had been infected in China, while more than 500 thousand people had been confirmed to have contracted the disease around the world. The main transmission of the virus is through respiratory droplets or by touching a contaminated surface. ${ }^{[1]}$ The symptoms of COVID-19 are lighter than those of the Severe Acute Respiratory Syndrome (SARS) and the Middle East Respiratory Syndrome (MERS), but the transmission ability is stronger than in those diseases. It is easily transmissible among humans, especially to older people with preexisting diseases. In China, the incidence rate of severe cases is about $10 \%$, and the mortality rate is about $2.1 \%{ }^{[2]}$

COVID-19 is a new coronavirus that can cause fever and coughing. It can rapidly develop into severe disease in some infected people, and may cause systemic inflammatory response syndrome (SIRS) and multiple organ dysfunction syndrome (MODS). A test for COVID-19 infection can be done now within 15-30 minutes. Therefore, the Chinese government suggested that all high-risk groups should be screened. However, according to the Chinese National COVID-19 Diagnosis and Treatment Guidelines (trial version 7), the following two conditions should be regarded as suspected cases: epidemiologic history plus two points and clinical manifestations; Three points with clinical manifestations. ${ }^{[2]}$

- Epidemiologic history: Travelled to or lived in Wuhan and its surrounding areas within the last 14 days; Had a contact with confirmed or suspected patients within the previous 14 days.

- Clinical manifestations: 1. Fever, cough, and other respiratory symptoms. 2. Imaging manifestations of pneumonia. 3. The total number of leukocytes is normal or decreased, and the percentage of lymphocytes is decreased.

For suspected patients to be completely cleared, they need to conform with the following two points: 1 . At an interval of at least 24 hours, two tests for novel coronavirus nucleic acids were negative for detection. 2. The novel coronavirus specific $\operatorname{lgM}$ and IgG antibodies remain negative seven days after the onset of symptoms. Therefore, it takes at least seven days for suspected patients to be completely excluded from being infected with COVID-19. ${ }^{[2]}$ How should anesthesiologists protect themselves if the suspected patients must go through an emergency operation? We report of anesthesia in a case of suspected COVID-19 patient with acute gastric hemorrhage.

\section{Case Description}

A 54-year-old man, $169 \mathrm{~cm}$ tall and weigh $56 \mathrm{~kg}$, was diagnosed with gastric ulcer by gastroscopy at the Luoyang Hospital after presenting with epigastric pain in January 2020. Black stool and epigastric pain occurred on March 10, 2020. The patient, however, did not pay enough attention and did not receive any treatment. Hematemesis occurred at the Beijing railway station in the afternoon on March 13, 2020. The patient was transferred to our emergency room by ambulance as soon as possible. According to the epidemiologic investigation, there was one case of confirmed COVID-19 infection in the village where the patient lived. He had direct contact with that person. The patient did not 
cough and had no fever. Routine blood examination showed that white blood cell (WBC) count was $8.08 \times 10^{9}$, of which lymphocyte accounted for $0.72 \times 10^{9}$. Chest CT showed that the two lungs had diffuse ground glass opacity with exudative lesions. A COVID-19 nucleic acid test on a throat sample was negative. The COVID-19 expert group concluded that the patient could not be completely ruled out of COVID19 infection. In the emergency room, the patient spat out a large volume of blood, and his blood pressure dropped. After surgical consultation, it was assumed that the patient had active gastric bleeding and needed an emergency operation. After reporting to the Beijing Center for Disease Control and Prevention (CDC), we prepared for surgery, treating the patient as a suspected case.

Preoperative preparation

We prepared an operating room for infected patients. Such an operating room should have a negative pressure airflow. The Anesthetists and other medical staff wore protective gear before contacting the patient. This included wearing washed clothes inside, N95 mask, disposable surgical cap, goggles or protective screen, C-class protective clothing outside, double latex gloves, and shoe covers. The patient was covered with a single operation sheet before being transported to the operating theater. The patient wore a medical protective mask without a breathing valve throughout the process.

The anesthesia process

On entering the operating room, the heart rate of the patient was 98 beats per minute (bpm), invasive arterial blood pressure was $88 / 54$ $\mathrm{mmHg}$, and peripheral oxygen saturation $\left(\mathrm{SpO}_{2}\right)$ was $96 \%$. Radial artery blood-gas analysis showed: $\mathrm{pH} 7.32$, hemoglobin $(\mathrm{Hb}) 78 \mathrm{~g} / \mathrm{L}$, and partial oxygen pressure $\left(\mathrm{PaO}_{2}\right) 76 \mathrm{mmHg}$. We removed the patient's $\mathrm{N} 95$ mask for oxygen inhalation before anesthesia induction, and connected an artificial nose to a anesthesia respiratory pathway in advance. The drugs of anesthesia induction were midazolam $2 \mathrm{mg}$, sufentanil $20 \mu \mathrm{g}$, etomidate $0.2 \mathrm{mg} / \mathrm{kg}$, and rocuronium $1.2 \mathrm{mg} / \mathrm{kg}$. When the patient lost consciousness, he was intubated with a visual laryngoscope. There was no noticeable coughing during intubation. The intubation equipment was immediately placed inside a double-layer sealed bag to be sterilized or discarded after the operation. Then the right internal jugular vein was punctured and catheterized for blood and fluid transfusion. Continuous mechanical ventilation was initiated with a tidal volume of $8 \mathrm{~mL} / \mathrm{kg}$ after general anesthesia was achieved. Target controlled infusion (TCl) of propofol and remifentanil was given for anesthesia maintenance and adjusted according to the Bispectral Index (BIS). Muscle relaxants were added according to the Train of Four (TOF), and the Stroke Volume Variation (SVV) was used to guide fluid therapy. During the operation, we transfused $800 \mathrm{~mL}$ of red blood cells and $400 \mathrm{~mL}$ of plasma. After the operation, the patient was transferred to the intensive care unit (ICU) of the isolation ward with the endotracheal tube in place.

Postoperative follow-up

The patient was extubated two hours after entering the ICU. Before extubation, two pieces of gauze were placed over his mouth to reduce the spatter caused by coughing. After extubation, the vital signs of the patient were stable, and he was transferred to the general surgical isolation ward 24 hours later. All doctors and nurses involved in the operation were placed under medical observation for two weeks, with their temperatures and other symptoms recorded by a person dedicated for the task. A second COVID-19 nucleic acid test of the patient was performed the next day, and fortunately, it turned out to be negative as well. The COVID-19-specific IgM and IgG antibody test seven days later was also negative. The COVID-19 expert group ruled out infection of COVID-19 on March 20, 2020, and all doctors and nurses involved in the case were relieved from medical observation.

\section{Discussion}

Anesthesia of suspected COVID-19 cases should be treated in the same way as that of confirmed COVID-19 cases. ${ }^{[2]}$ Preventive measures in emergency operations should be carried out according to the guideline for severe infectious diseases, while non-emergency operation should be postponed until after the COVID-19 is cured. ${ }^{[3]}$ Although the rapid COVID-19 nucleic acid test of the patient was negative, it was necessary to perform a second test 24 hours later or to check for specific antibodies in the serum seven days later. Therefore, this patient had to be treated as a suspected COVID-19 patient.

Before anesthesia, a special infection treatment-ready operating room with a buffer zone should be prepared. The operating room should be closed before the operation to create a negative pressure (- $5 \mathrm{~Pa}$ or less). All the staff members involved in the operation should wear protective equipment before contacting such patients, including wearing washed clothes inside, a medical protective mask, a disposable surgical cap, goggles or a protective screen, c-class protective clothing outside, double latex gloves, and shoe covers. As is well known, the main transmission of COVID-19 among people is through respiratory droplets. It can easily be transmitted in relatively closed environments in which people are exposed to a high concentration of aerosols.[4] Endotracheal intubation was necessary to reduce the spread of air and droplets from the patient's mouth and nose. However, the risk to anesthetists is extremely high during the intubation process. According to the experience gained from SARS prevention, personal protection should be strengthened, including wearing N95 masks and protective

Page $3 / 5$ 
screens. These can effectively filter out $98 \%-100 \%$ of particles above $0.3 \mu \mathrm{m}$, and prevent possible contamination of the cornea and skin by aerosols.[5] The key point of endotracheal intubation in such cases is to avoid splashing of secretion by coughing.[6] Therefore, we should increase the dosage of neuromuscular blocking agents. We used rocuronium at 3-4 times the 95\% effective dose (ED95) for the induction of this patient. The patient should be sent to the ICU in an isolation ward after operation, and the endotracheal tube should be removed only after the vital signs are stable and the anesthetic drugs have been fully metabolized. Before extubation, pieces of gauze should be used to cover the patient's mouth to reduce the spatter caused by coughing. An artificial nose is recommended for use during such operations as it can effectively prevent the anesthesia machine from becoming contaminated by bacteria and viruses. It should, however, be replaced every three to four hours.[7] According to the experiences gained during the SARS and MERS epidemics, a pulmonary protective ventilation strategy is recommended for asymptomatic or mildly infected patients undergoing intraoperative mechanical ventilation. Such ventilation includes lower tidal volume and a suitable positive end-expiratory pressure (PEEP).[8] Individualized ventilation strategy should be planned for severe or critical infected patients based on changes in their lung functions. The patient should be covered with a single operation sheet before being transported to the operating room and should be moved by a dedicated elevator. It is suggested to evacuate the transfer route in advance to reduce the exposure of unrelated personnel. Disposable anesthetic supplies should be used as much as possible, including endotracheal tube, laryngoscope, dental pad, suction tube, suction device, mask, anaesthetic screw tube, and so on.[9] After use, these should be placed in a special double-layer garbage bag marked with a visible mark for proper disposal after the surgery. Non-disposable equipment should be properly washed and sterilized according to the disinfection standard for equipment that was contaminated by patients with particular infections. The operating room, corridor, elevator, and isolation ward should all be washed and sterilized according to the guideline. Finally, an expert group for COVID-19 should be established during the epidemic period. This group includes surgeons, nurses, and anesthesiologists. Each hospital should formulate guidelines for the screening and treatment of infected or suspected emergency patients, according to its situation.

\section{References}

1. Respiratory care committee of Chinese Thoracic Society. Expert consensus on preventing nosocomial transmission during respiratory care for critically ill patients infected by 2019 novel coronavirus pneumonia[J]. Chin J Tubere Respir Dis. 2020 Feb 20;17(0):E020.

2. Health Emergency Office of National Health Commission of the PRC. Update on the diagnosis and treatment of 2019 Novel coronavirus pneumonia (COVID-19) $₫ T$ The Seventh Edition $\otimes$ [Article in China]. http://www.nhc.gov.cn/yzygj/s7653p/202003/46c9294a7dfe4cef80dc7f5912eb1989/files/ce3e6945832a438eaae415350a8ce964.pdf.

3. Perioperative Infection Control Society of Chinese Society of Cardiothoracic and Vascular Anesthesia, PLA Society of Anesthesiology and Resuscitation. Recommendation for perioperative infection control in patients with 2019 Novel coronavirus pneumonia (COVID-19) [J]. Preoperative Safey and Quality Assurance.2020,4(2):63-66. [Article in China] . https://mp.weixin.qq.com/s/TKBKhuRsoOe9XailOfp5Ng.

4. Jin YH, Cai L, Cheng ZS, Cheng H, Deng T, Fan Y-P, et al. A rapid advice guideline for the diagnosis and treatment of 2019 novel coronavirus (2019-nCoV) infected pneumonia (Full version) [J]. Journal of New Medicine. 2020;30(1):35-64. [Article in China]

5. Bo LL, Wan XJ, Bian JJ, Deng XM. COVID-19 patients undergoing tracheal intubation: a literature review based on the airway management in SARS patients[J] . Chin J Anesthesiol,2020,40(02): 129-132. DOI: 10.3760/cma.j.issn.0254-1416.2020.0002

6. Bowdle A, Munoz-Price LS. Preventing Infection of Patients and Healthcare Workers Should Be the New Normal in the Era of Novel Coronavirus Epidemics. Anesthesiology. 2020. doi:10.1097/ALN.0000000000003295.

7. Ryan AJ, Webster CS, Merry AF, Grieve DJ. A national survey of infection control practice by New Zealand anaesthetists[J]. Anaesth Intensive Care 2006;34:68-74.

8. Julie D ,Robin G, Jason K. Middle East Respiratory Syndrome and Severe Acute Respiratory Syndrome: Current Therapeutic Options and Potential Targets for Novel Therapies[J].Drugs. 2017;77(18): 1935-1966.

9. Association of Anaesthetists of Great Britain and Ireland (AAGBI). Infection Control in Anaesthesia[J]. Anaesthesia 2008;63:1027-1036.

\section{Declarations}

Ethics approval and consent to participate This trial was approved by the sixth medical center of PLA general hospital (No. 20200128, 22 March 2019) and written informed consent was obtained from all participants.

A statement on participant consent The patient agreed to allow the researcher(s) to take photographs of me and grant permission for these to be used by the researcher(s) and their project partners in publications, press articles and websites, exclusively for non profit-making purposes $\llbracket$ and signed informed consent. 


\section{Figures}

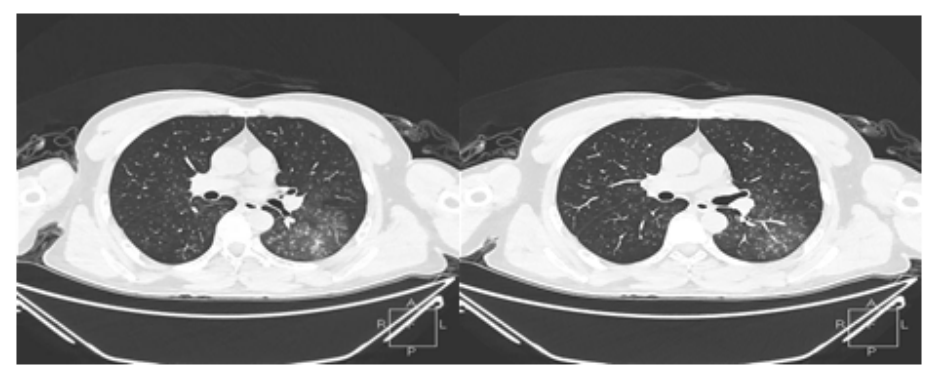

\section{Figure 1}

CT of the patient before the operation

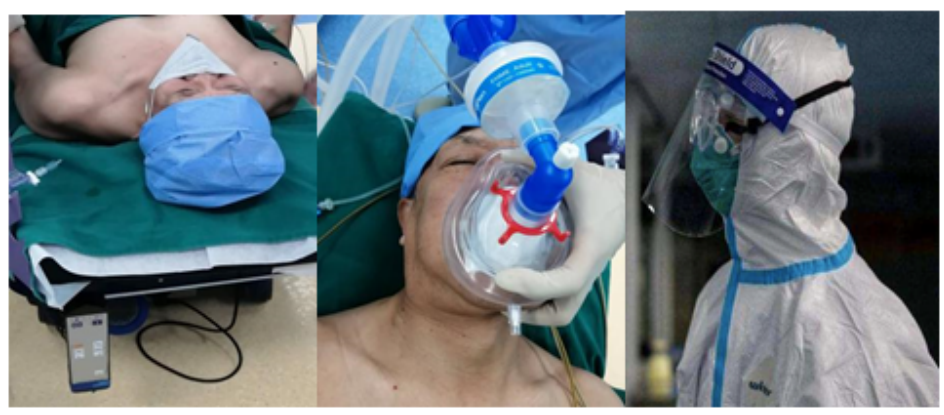

Figure 2

Protective measures during anesthesia induction and intubation 